\title{
Optical budgeting for LUVOIR
}

Paul A. Lightsey, J. Scott Knight, Matthew R. Bolcar, Lee D. Feinberg, William L. Hayden, et al.

Paul A. Lightsey, J. Scott Knight, Matthew R. Bolcar, Lee D. Feinberg, William L. Hayden, Michael J. Eisenhower, Sang C. Park, "Optical budgeting for LUVOIR," Proc. SPIE 10698, Space Telescopes and Instrumentation 2018: Optical, Infrared, and Millimeter Wave, 1069813 (6 July 2018); doi: $10.1117 / 12.2312256$

SPIE Event: SPIE Astronomical Telescopes + Instrumentation, 2018, Austin, Texas, United States 


\title{
Optical budgeting for LUVOIR
}

\author{
Paul A. Lightsey*a, J. Scott Knight ${ }^{\text {a }}$, Matthew R. Bolcar ${ }^{\mathrm{b}}$, Lee D. Feinberg ${ }^{\mathrm{b}}$, \\ William L. Hayden ${ }^{b}$, Michael J. Eisenhower ${ }^{c}$, Sang C. Park ${ }^{\mathrm{c}}$ \\ ${ }^{a}$ Ball Aerospace \& Technologies Corp., 1600 Commerce Street, Boulder, CO, USA 80301; \\ ${ }^{\mathrm{b}}$ NASA, Goddard Space Flight Center, Greenbelt Road, Greenbelt, MD, USA 20771; \\ ${ }^{\mathrm{c}}$ Harvard-Smithsonian Center for Astrophysics, 60 Garden Street, Cambridge, MA 02138
}

\begin{abstract}
Future large astronomical telescopes in space will have architectures that will have complex and demanding requirements to meet the science goals. The Large UV/Optical/IR Surveyor (LUVOIR) mission concept being assessed by the NASA/Goddard Space Flight Center is expected to be 8 to 16 meters in diameter, have a segmented primary mirror, active control, and be diffraction limited at a wavelength of 500 nanometers. The optical stability is expected to be in the picometer range for minutes to hours. Architecture studies to support the NASA Science and Technology Definition teams (STDTs) are underway to evaluate systems performance. A wave front error budget has been developed to help define the technology needs and assess performance. The budget includes both spatial and temporal domain aspects for the active, adaptive and passive elements in the optical design.
\end{abstract}

Keywords: Space Telescope, optical error budgeting, Segmented telescopes, Point Spread Function, Strehl ratio, encircled energy, wave front error, spatial frequency, line-of-sight jitter, coronagraphy.

\section{INTRODUCTION}

The science goals for future astronomical missions will require large deployable space telescope architectures similar to the James Webb Space Telescope (JWST). One such mission currently being assessed by NASA is LUVOIR, a large UV, optical, IR capable observatory ${ }^{1,2}$. A Science and Technology Definition Team (STDT) has been formed and is underway in defining the science goals and architecture concepts to achieve astrophysics and exoplanet science goals over the spectral range from the UV to NIR. LUVOIR is expected to be 8 to 16 meters in diameter, have a segmented primary mirror and be diffraction limited at a wavelength of 500 nanometers. The Advanced Technology Large Aperture Space Telescope (ATLAST) ${ }^{3}$ study provided an example of a LUVOIR type segmented telescope concept, shown in Figure 1 along with JWST and LUVOIR concepts.
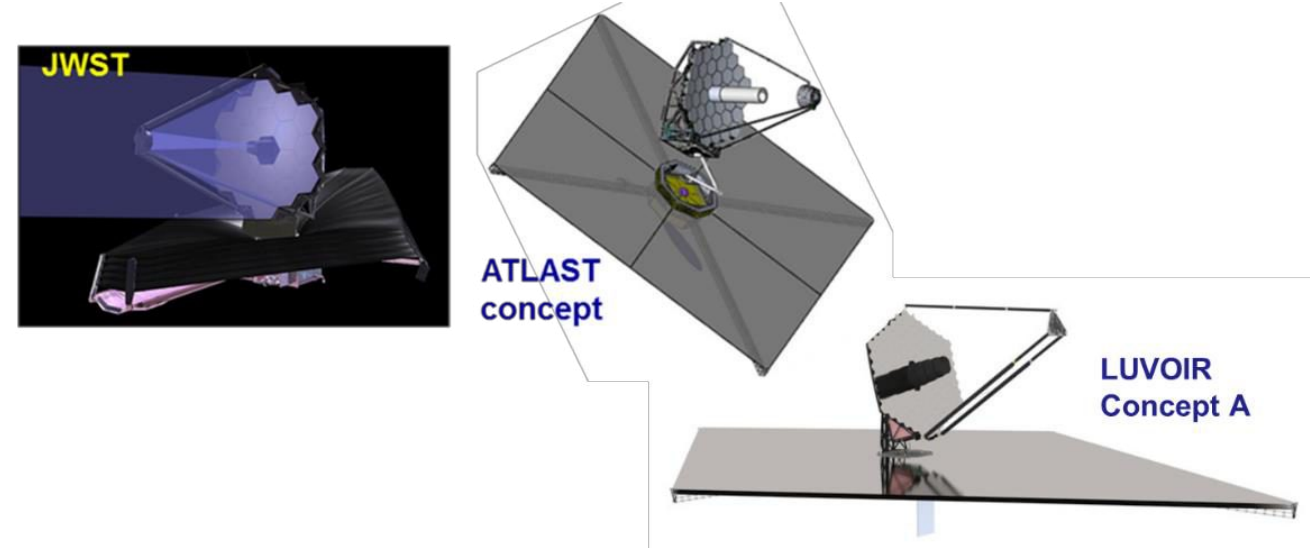

Figure 1. Examples of large segmented space telescope designs ranging from 18 to 120 hexagonal PM segments.

The operation at the shorter UV wavelengths and the needs of high contrast coronagraphy require a challenging evolution from the JWST optical budget. The coronagraphy requires precise control of the WFE to compensate for

Space Telescopes and Instrumentation 2018: Optical, Infrared, and Millimeter Wave, edited by Makenzie Lystrup,

Howard A. MacEwen, Giovanni G. Fazio, Proc. of SPIE Vol. 10698, 1069813

(C) 2018 SPIE · CCC code: 0277-786X/18/\$18 - doi: 10.1117/12.2312256 
the inherent diffraction pattern of the aperture shape and segmentation and to control the PSF pattern to allow high contrast at small angular separation from the PSF centroid. The stability of the precision WFE control must be maintained to the picometer range for observational time intervals ranging from minutes to hours.

\section{POINT SPREAD FUNCTION CHARACTERISTICS}

The fundamental PSF diffraction pattern is dominated by the shape of the aperture. The traditional ideal shape is an unobscured circular aperture giving an Airy pattern for the PSF. For an on-axis Cassegrain type of telescope, the Secondary Mirror (SM) produces a central obscuration with a circular annulus aperture pattern. The diffraction for the annulus is nearly an ideal Airy pattern with some departure depending on the obscuration ratio. As telescopes have become larger and evolved to segmented apertures, the shape of the tiling pattern for the segments becomes the dominate factor in determining the shape of the diffraction pattern. The diffraction pattern for perfect (zero wavefront error) segmented apertures have been calculated ${ }^{4,5}$ with results shown in Figure 2

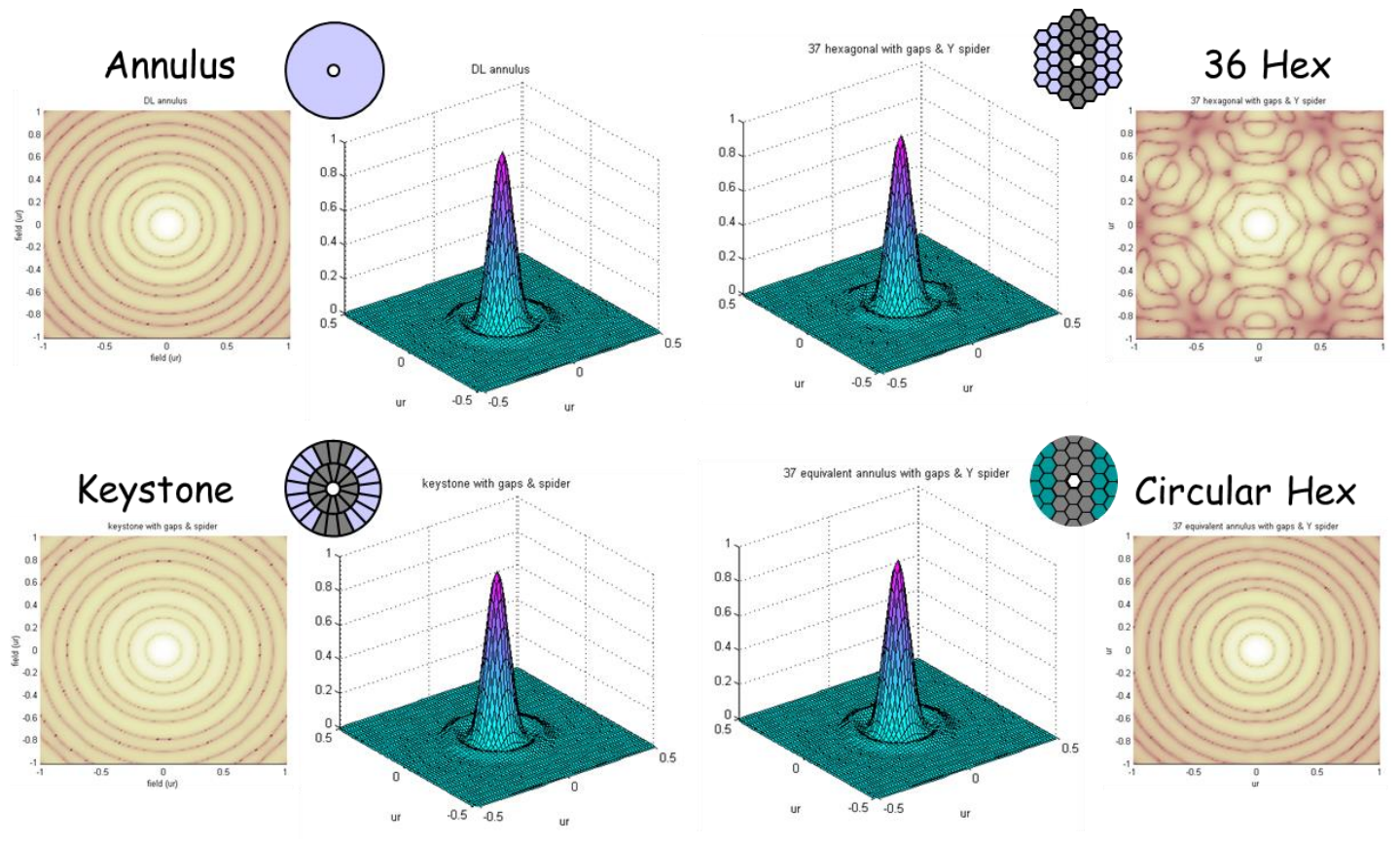

Figure 2. PSFs shown for a monolithic annulus aperture compared to circular Keystone, circularized 36 hexagons, and full 36 hexagons. Log stretched contour plots and linear perspective plots shown for each.

These results show that the dominate factor in the shaping of the PSF is the perimeter of the aperture. If a circular mask is used to eliminate the serrated edge of the hexagonal pattern, the differences between a monolithic annulus, a circular keystone tiling pattern, or the circularized hexagonal pattern are small. For gaps that are less than $0.1 \%$ of the aperture diameter, the effect of their diffraction is small compared to diffraction from the aperture shape and the struts supporting the SM.

The telescope will not have perfect zero WFE, so the task at hand is to determine the metrics that provide the measure and insight into the PSF quality to achieve the science goals. And furthermore, how to convert those metrics into measures that provide engineering guidance to fabrication, alignment, and verification of the optical system.

\section{METRICS TO ASSESS PSF QUALITY}

The PSF as a complete measure of image quality is useful for simulating science data acquisition by convolution of the PSF with expected scenes. However, during the design, fabrication, integration, and testing of an optical system, simpler merit functions are desired. There are several features of the PSF that lend themselves to being characterized by a simpler merit function. A few of these simpler scalar number metrics that have been used extensively in the past have been Strehl Ratio, encaptured energy (encircled or ensquared), full width half maximum (FWHM), and related wave front error (WFE )metrics such as rms WFE. William Wetherell ${ }^{6}$ has pointed out that image quality analysis has 
been beset by "unimania" - the belief that highly complicated processes can be compared fully and accurately using a single one-real-number merit function. Thus, there has been an over reliance on these single value merit functions. To avoid this problem, a combination of single number scalar metrics will be used to collectively control different aspects of the PSF that affect the ability to achieve the desired science objectives. The different metrics have different utility. The utility of several common metrics are as follows.

WFE (rms): Gives a broad general measure of PSF quality. It is the square root of the variance of the wave front error over the pupil.

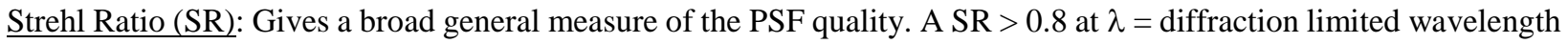
used as a common indicator of diffraction limited performance. SR is directly related to the statistical variance of the WFE (or optical path difference, $\sigma_{\text {opd }}^{2}$ ) as given by

$$
S R_{w f e}=e^{-\left(2 \pi^{\sigma_{\text {opd }} /} / \lambda\right)^{2}} .
$$

The SR can also be calculated for image motion based on the variance of the line-of-sight pointing during the image exposure. The calculation is given by

$$
S R_{i m}=\left[1+\left({\sigma_{i m x}}^{2}+\sigma_{i m y}{ }^{2}\right) \cdot(\pi D / 2 \lambda)^{2}\right]^{-1},
$$

where $\sigma_{\text {imx }}{ }^{2}$ and $\sigma_{\text {imy }}{ }^{2}$ are the variance of the image motion in two orthogonal axes in the image plane. The SR depends on the relative scaling of the rms image motion to $(\lambda / \mathrm{D})$ where $\mathrm{D}$ is the diameter of the entrance aperture.

An equivalent $\sigma_{\mathrm{opd}}{ }^{2}$ WFE can be calculated for $\sigma_{\mathrm{imx}}{ }^{2}$ and $\sigma_{\text {imy }}{ }^{2}$ by equating the SRs, allowing image motion to be included as an allocation within a WFE budget.

Encircled Energy Fraction (EEF): Fraction of total PSF energy captured within a specified radius of the center of the PSF for a specified $\lambda$. Choice of $\lambda$ and radius can be used to control specific spatial frequency content of WFE. EEF also has utility as a photometric aperture measure of radiometric performance including comparison of image to background (stray light).

Image Sharpness: Captures broad general quality of PSF and is useful as an alternative to the photometric aperture for radiometric measures. The sharpness expressed as the inverse of an effective solid angle size for the PSF as given by:

$$
\text { sharpness }=\frac{1}{\Omega_{b k g r}}=\frac{\iint d \Omega \cdot(P S F)^{2}}{\left(\iint d \Omega \cdot P S F\right)^{2}} .
$$

For the discrete pixelization of the focal plane, the sharpness can be expressed in terms of pixels rather than solid angle as given by:

$$
\text { sharpness }=\frac{1}{N_{b k g r}}=\frac{\sum_{i} \sum_{j} P S F_{i j}{ }^{2}}{\left[\sum_{i} \sum_{j} P S F_{i j}\right]^{2}} .
$$

FWHM: This has utility of capturing the effective sharpness of the PSF, and for reasonably circular symmetric PSFs, is an alternative to image sharpness that is more directly determined.

PSF anisotropy: Utility is to manage the shape of the PSF to reduce asymmetries that decrease the ability to measure anisotropy in galactic shapes from gravitational lensing. The anisotropy is calculated using second moments of the PSF weighted by a Gaussian profile shape for the galaxy of interest.

Contrast Ratio: Utility is for use in high contrast imaging, especially for managing performance of coronagraphs utilizing wave front control to reshape the PSF creating dark regions near the core of the PSF. The specification is given as the ratio of the PSF at a given angle from the centroid to the peak PSF value. The interest is for applications 
such as exoplanet imaging. The contrast ratio will depend on both the pupil shape and amplitude function and WFE. For WFE budgeting purposes, the intent is to convert from the single scalar metric of contrast ratio to specific metrics that address: 1) the sensitivity of the contrast ratio to the WFE within specific spatial frequency modes use to control the PSF speckle, 2) magnitude of WFE control resolution necessary, 3) range of WFE control needed, 4) temporal stability and or temporal control bandwidth required. The budget design accommodates a nested layered, or hierarchy approach to controls and will be adapted to the specific coronagraph architecture options. This is one area of the budget that will rely on simulations and analysis to better define and validate the budgeting approach. The development will be an iterative process.

\section{LARGE SEGMENTED DEPLOYABLE APERTURES}

Budgeting for optical performance for large segmented deployable telescopes depends on Wave Front Sensing and Control (WFSC) with the following staged levels of optical tolerances.

- Tolerances following deployments

- Tolerances following initial active alignments and phasing of the optical system using WFSC

- Tolerances for nested active control for different spatial domains with differing temporal bandwidths

The nested controls will include those internal to telescope level control and controls which affect all instrument fields, and those within coronagraph instrument that may compensate errors for the end-to-end optical train that primarily affect the coronagraph imagery. Some architectures may include some feedback control to the telescope that would affect all instruments.

Each stage has a parallel budget for active adjustment range, aka "Actuator Range Budget".

The optical budget for (WFE) must also be compatible with other optical alignment metrics such as pupil alignments and clearances of ray paths to avoid obscuring or vignetting beams.

The budget must accommodate multiple instruments and instrument modes. The budget needs to assure that interactions among the instruments during the active control processes are understood and accounted.

Coronagraphy drives unique requirements into the optical budget. The diffraction pattern and static WFE of the telescope presents the range over which the coronagraph active controls (DM) must operate to create a dark hole in the PSF in regions between the inner and outer working angle, aka speckle control. Short term stability sets the temporal bandwidth of adaptive controls to achieve static performance. Additional contrast is gained using speckle subtraction based on Differential Imaging (referenced or angular). This places longer term temporal stability requirements on the system. The sensitivity of contrast to WFE varies with spatial frequency content ${ }^{7}$, so the optical budget will need to account for combination of temporal and spatial frequency characteristics to effectively budget to meet contrast performance, and be structured to account for where control is implemented

The spatial frequency domains for budgeting are set by the nature of the active control (see Figure 3). The Strehl Ratio for the system will depend on total WFE, where Encircled Energy will depend on spatial frequency content scaled by the ratio of the encircling radius to $(\lambda / \mathrm{D})$. The low spatial frequency domains will rely on active alignment of the SM and global (correlated) movement of the PM segments to effect spatial order shaping and alignment of the PM. Mid spatial frequency domain will rely on the ability to adjust PM segments individually. For coronagraphy, the instrument may have and internal deformable mirrors (DMs) to provide additional control. The high spatial frequency domain is controlled during manufacture of the individual mirrors and is not actively controlled during flight. 


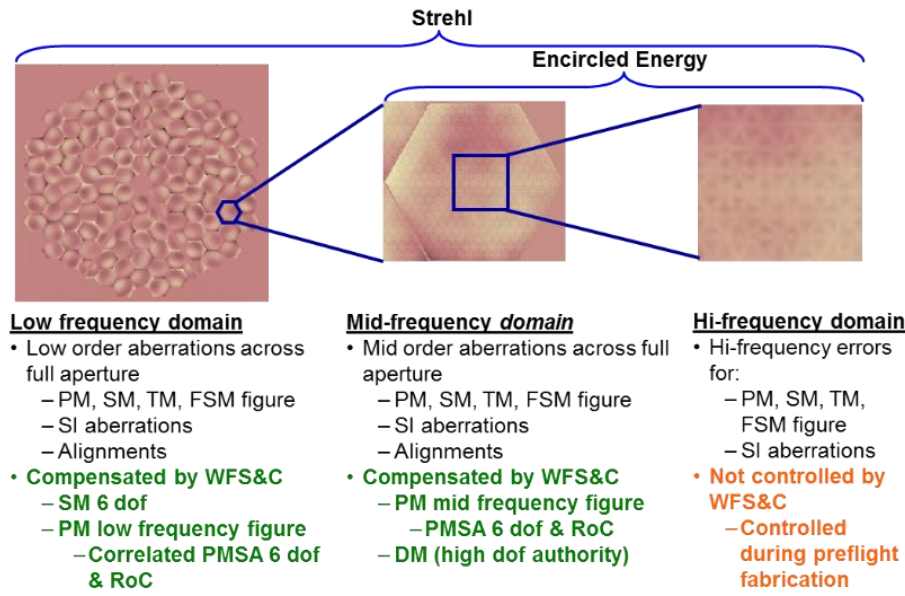

Figure 3. The spatial frequency domains categorized by controllability.

There will also be utility in providing multiple temporal frequency domains within the budget structure.

Long time scales: These will be for the time interval between periodic updates of the static WFE. This process will likely use Wavefront Sensing and Control (WFSC) at a cadence of several days to weeks.

Medium time scales: These are set by duration of the variability of the thermal environment in response to changes in observational pointing or other operational activities. These time scales will be from minutes to days. The budget is designed to handle tolerances for both active thermal controls and passive controls.

Short time scales: These cover the time intervals for active systems with adaptive controls to compensate changes and will range from sub second to minutes. The types of control include opto-mechanical sensing and control, Line-ofsight and Low Order Wave Front controls, and active vibration isolation.

Very short time scales: These are time scales that correspond to frequencies above control bandwidths. This temporal domain is to account for uncompensated dynamics greater than a few $\mathrm{Hz}$.

\section{BASIC STRUCTURE OF BUDGET}

The budget is designed to show the total budget for the telescope and an instrument, and is switchable among the different conditions to be considered in evaluating the budget as shown in Figure 4. The choices for conditions are a combination of:

- Instrument to evaluate: HDI, LUMOS, ECLIPS, ONIR, or POLLUX

- Wave Front Sensing method to be used for basic alignment and phasing: Single Field Point; Single Instrument/Multi-field points; Multi Instrument/Multi-field points

- Moving Target (or not)

- Time domain: time interval over which the stability values apply: short; medium; long

- Status: selects whether the budget is for the baseline design, or does it include known liens and accepted opportunities against the design, or potential threats and opportunities.

The latter choices for status are used later in the life cycle of the program for risk management. 


\begin{tabular}{|lr|}
\hline Conditions & \\
instrument & ECLIPS \\
WFS & MIMF \\
moving target & no \\
time domain & medium \\
status & base \\
\hline
\end{tabular}

Figure 4. Conditions box in budget that has switches for selecting instrument, WFS technique, whether the target is moving or not, time domain, and budget status relative to risk posture.

The basic structure of the budget is a tree showing the branching and subsequent flow down of allocations. The branches spread out to the constituent contributors as shown in Figure 5. Each box in the budget tracks the allocation (Alloc), the "best estimate" prediction (pred), and the uncertainty in that prediction (unc). At the Observatory level, the budget explicitly allocates a portion of the top-level requirement to Management Reserve. This is allocated for program control and risk management. The boxes identified as margin shows the margin of the aggregated roll up of the lower level sub allocations compared to allocation, and the roll up of the combined prediction and uncertainty compared to the allocation. Margin for management of risks at the lower levels is embedded within the lower level allocations, while the aggregate roll-up of $\mathrm{P}+\mathrm{U}$ shows margin of the predicted performance with uncertainty against the higher-level allocation. [Note that at early stages of development, the entries for prediction and uncertainty are set to zero, and only the allocations are entered. As the program progresses, analysis of the proposed design is used to populate the prediction and uncertainty, and later they are replaced by verification values.]

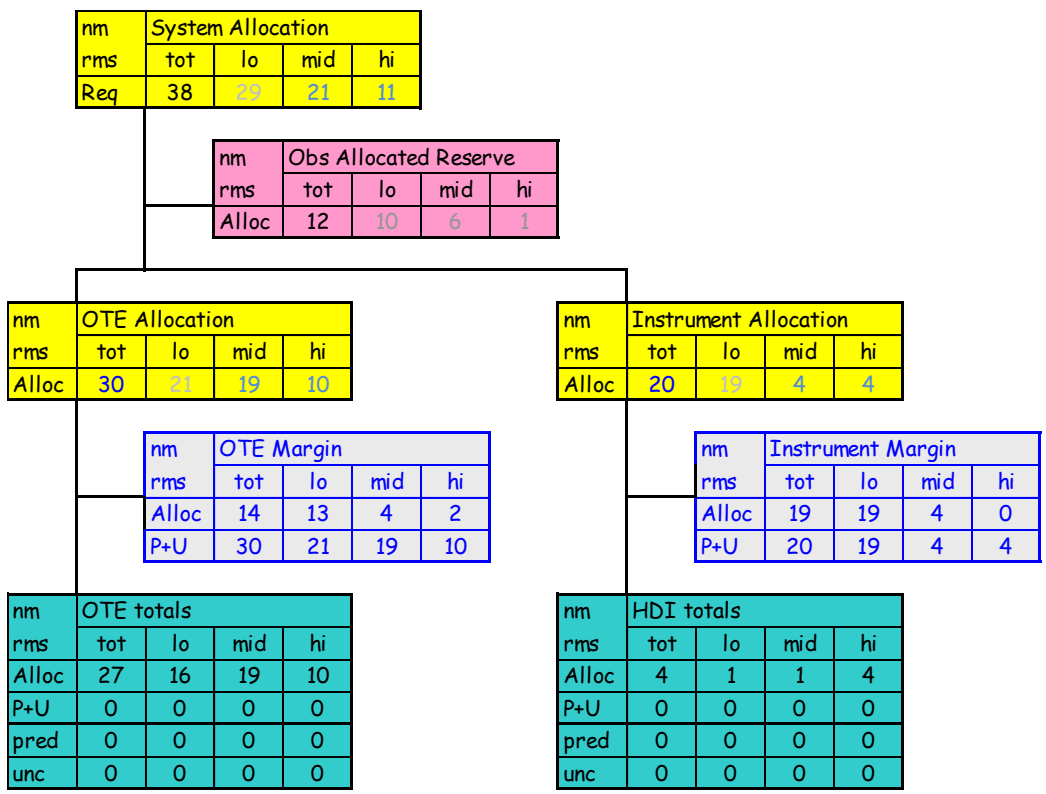

Figure 5. Top level allocation divided between telescope and instrument constituents. Allocated Reserve and Margin tracking included.

Figure 6 shows the budget structure arranged in a matrix pattern to distinguish between hardware constituents (in the vertical columns) and the categories of the phenomena being tracked (in the horizontal rows). The hardware splits at the top level between telescope and instruments, and at subsequent lower levels branches into more detailed components. For example, the telescope would divide into mirrors (PM, SM, TM, FSM) and integrating structures (Aft optical bench, PM backplane, SM support structures). Likewise, the instrument would divide into internal components and bench. The horizontal rows break into three top level categories: 1) residual following completion of a WFSC update, 2) stability between updates, 3) image motion. 

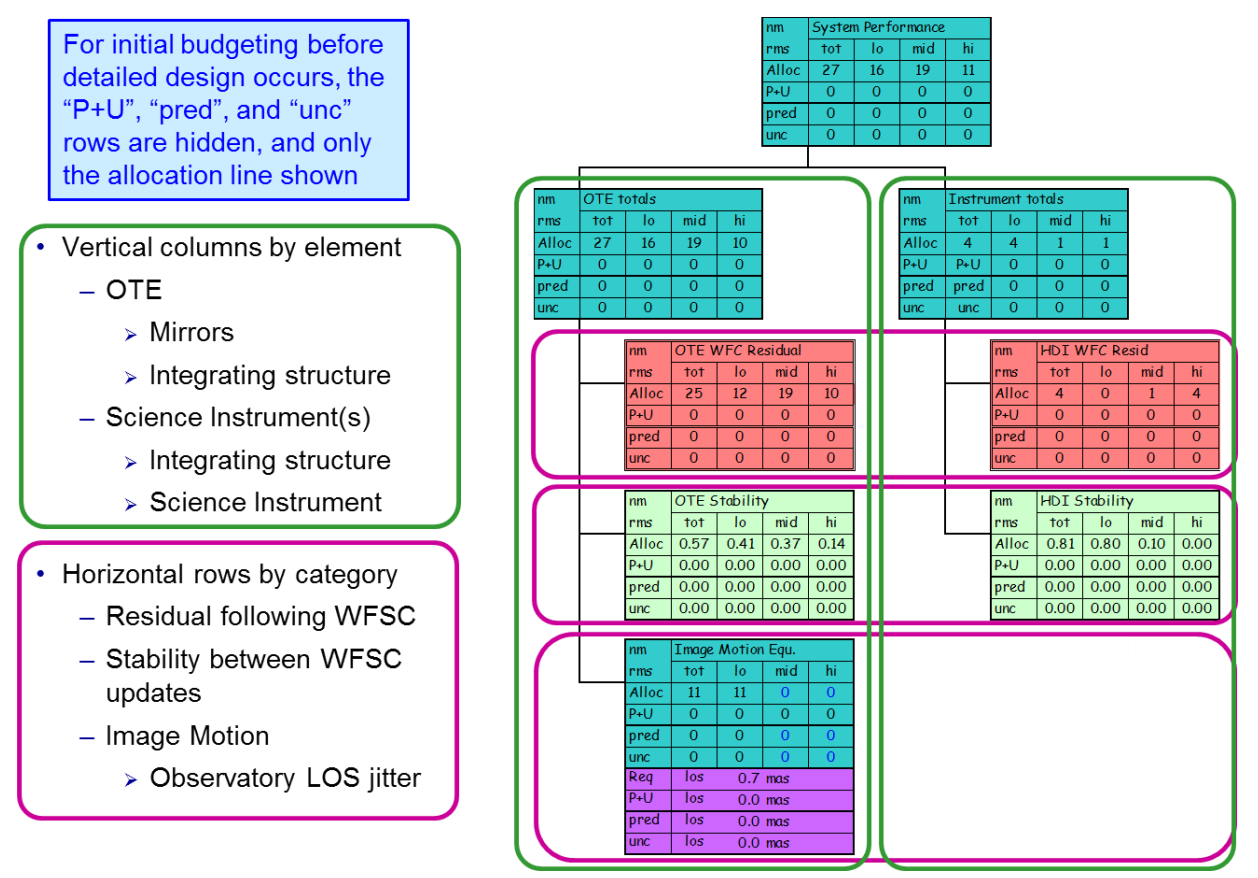

Figure 6. Highest level system budget arranged in a matrix fashion with components arranged by columns, and phenomena arranged by rows. Aspects of image motion cross component boundaries and is lumped under the telescope.

How entries in the budget mathematically combine is determined by the nature of the process. For combinations of a small number of entries that are not mutually orthogonal, the terms would linearly add; for example, alignment errors in a single degree of freedom. Note that the predictions would add, but the uncertainties would rss if they were statistically random errors. If the wavefront errors from differing boxes have independent and varying spatial content (different Zernike like polynomials), the results would rss. Where feasible, understanding the probability distribution for the underlying errors in the uncertainty line will help in later analyses and verification.

\section{WAVE FRONT SENSING AND CONTROL RESIDUAL}

The residual following wave front sensing and control is similar to the static wave front error for traditional systems. However, it relies on active WFSC processes to reach the final state. The WFSC process is done as part of the initial deployment and commissioning of the telescope, and then at relatively infrequent intervals after that for maintenance with a cadence of days to weeks. Higher frequency active control to compensate errors will be discussed as part of the stability allocations in Section 7. The basic portion of the residual error includes the effects of compensation used during Integration and Test before flight and from active compensation during the WFSC process during flight. There are then additional errors associated with the inflight WFSC process shown in Figure 7.

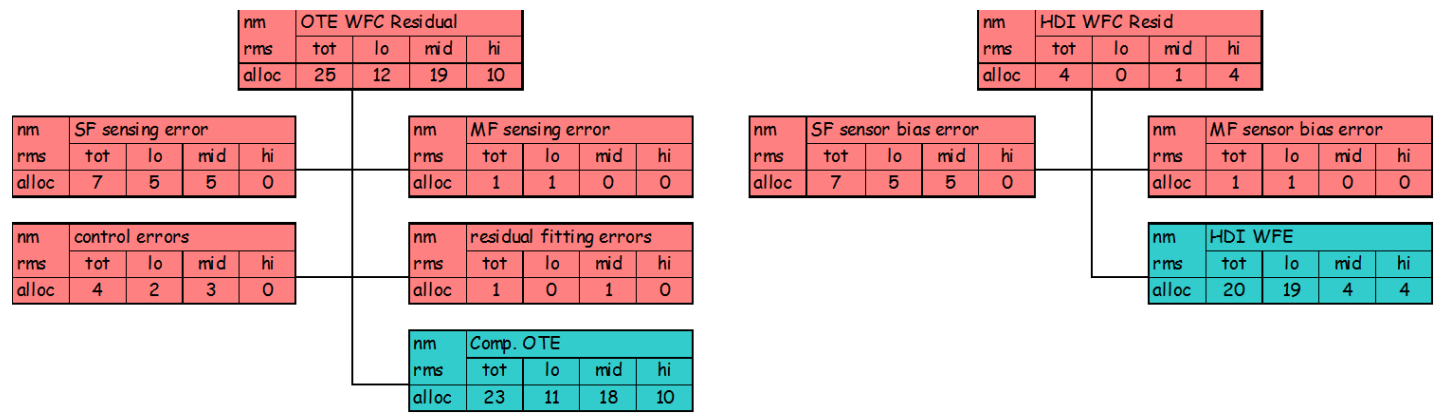

Figure 7. The contributors to the residual WFE following the WFC process. The sensing and control errors for OTE are combined with the residual compensated WFE of the OTE. For the science instruments, the additional contributions combined with the compensated residual are the sensor bias terms. The High Definition Imager (HDI) instrument is shown for illustration. 
For the telescope, the additional WFSC process errors are: 1) Single Field Point sensing error, 2) Multi-field Point sensing error, 3) control error, and 4) residual fitting error. The Single Field Point sensing error is how well the WFSC process can measure the WF at the single field point used to control low and mid spatial frequency errors within the telescope. The Multi-Field Point sensing error is an amalgam of how well the WF can be measured at multiple field points. These measurements are used to determine the alignment control of the PM and SM to eliminate the low spatial frequency field dependent errors in the telescope. In addition to sensing, there are the control errors consisting of the controllability and command resolution errors for the mirror actuation, and the fitting error between the smoothly varying wave front aberration to be corrected and the piecewise fitting of the segments having fixed surface figure.

For the instruments, the additional WFSC process errors are the result of bias errors in the instruments. To optimize the telescope at the single field point using imagery in a single instrument requires a priori knowledge of the instrument $\mathrm{WF}$ at that field location. The known instrument WF, or bias, is removed from the solution to determine the corrections for the OTE WF. Errors in the known WF bias of the instrument will be inadvertently compensated by the telescope, making the end-to-end WFE for that instrument channel better, but introducing error into the telescope which will add to the end-to-end WFE in other instrument channels. Likewise, calibration, or knowledge errors in the field dependent WF at the multi-field locations results in a multifield bias error. This error would introduce a field dependent error into the telescope that will affect the instruments differently depending on their location in the overall OTE field of view.

\subsection{TELESCOPE ELEMENT}

The budgeting of the OTE residual error follows a traditional approach as shown in Figure 8. This approach has three components. The first is the design residual, that is, the allocation for the optimized design prescription, even if the system was built perfectly. Then there are allocations for the alignment tolerances of the mirrors. The alignment allocations account for both individual independent alignment errors, as well as correlated alignments resulting from alignment of subsystem optical benches to the OTE (e.g. aft optics bench and the bench integrating the individual science instruments). And finally, the allocations for the individual surface figure errors of the mirrors. The global PM figure includes allocations for the 6 dof pose and RoC adjustments of the mirror segments in addition to the figure polished into individual segments. The SM, TM, and FSM figure errors are partially compensated by the active PM segment 6 dof and RoC control. The budget shows the residual errors for those mirrors after the WFSC process has partially compensated the fabrication figure errors.

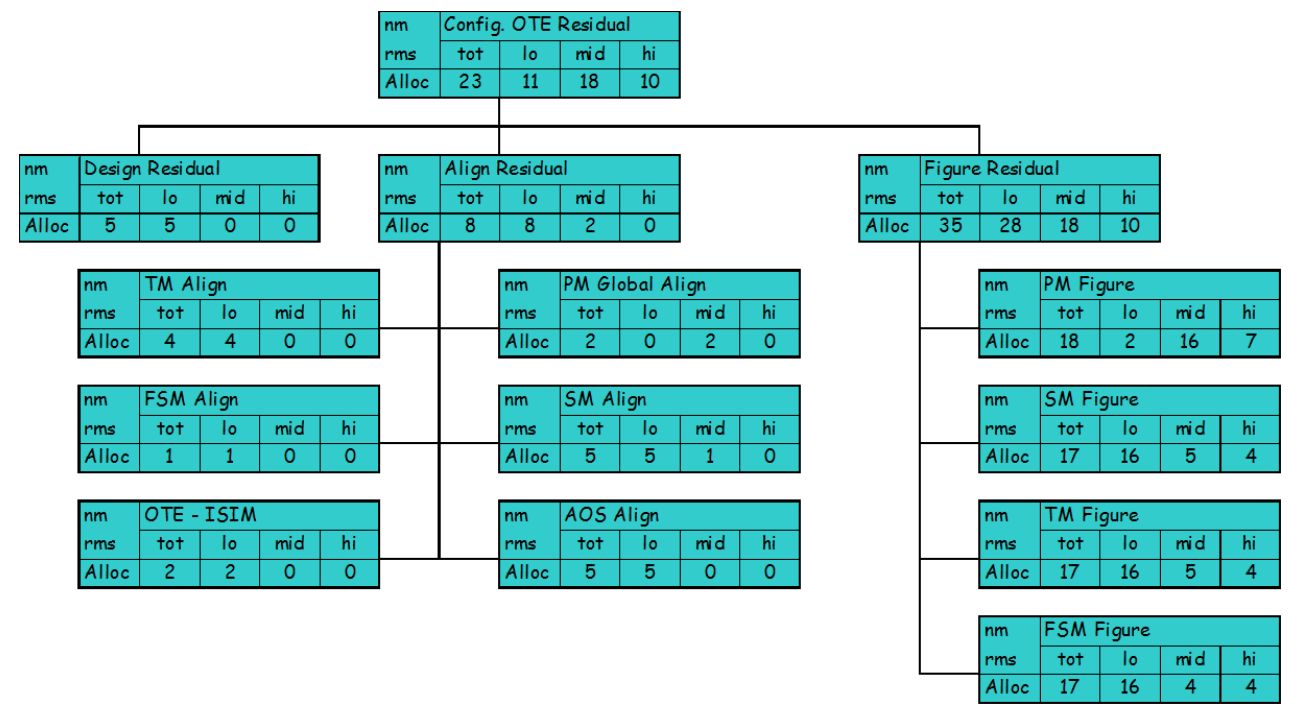

Figure 8. The OTE budget structure showing the allocations to: the design residual from the perfectly built optical prescription; the less than perfect alignment; and surface figure fabrication errors. The partial compensation of the figure errors in SM, TM, and FSM using active control of the PM segments is included in the budget. 
Often, the traditional methodology includes a separate allocation for the metrology for aligning and fabricating the mirrors. However, the method used in this budget has those metrology error embedded in the uncertainty portion of each allocation.

\subsubsection{PM}

The lower level details for the PM are shown in Figure 9. The PM figure is divided into three legs.

The first is for the post WFSC corrected errors for the individual segment figure. Errors in the curvature of the segment (RoC error) can be corrected by the RoC actuator, but the flexure mounting induces small amounts of higher spatial frequency distortions into the surface. Likewise, low order aberrations may be actively compensated. For example, for a semi-rigid segment architecture, astigmatism error in the fabricated mirror segment can compensated by decentering and/or clocking of the segment. But again, there is a small residual coma error and slight induced distortions from the flexures. If there is higher authority of control of the segment figure, this box would capture the residual errors following actuation. The bulk of post WFSC error is from the fabricated surface figure errors for the higher spatial frequency surface modes that cannot be corrected or compensated by the degrees of freedom available to the WFSC process. The lower level details to be considered as the budget is developed are: mirror segment fabrication and assembly (including conic error), backplane integration, I\&T and Launch shifts, operating temperature uncertainty, and Beginning of Life (BOL) to End of Life (EOL) changes. The BOL to EOL changes include long term stress relaxation and deformations resulting from the flight environment such as micrometeoroid impacts.

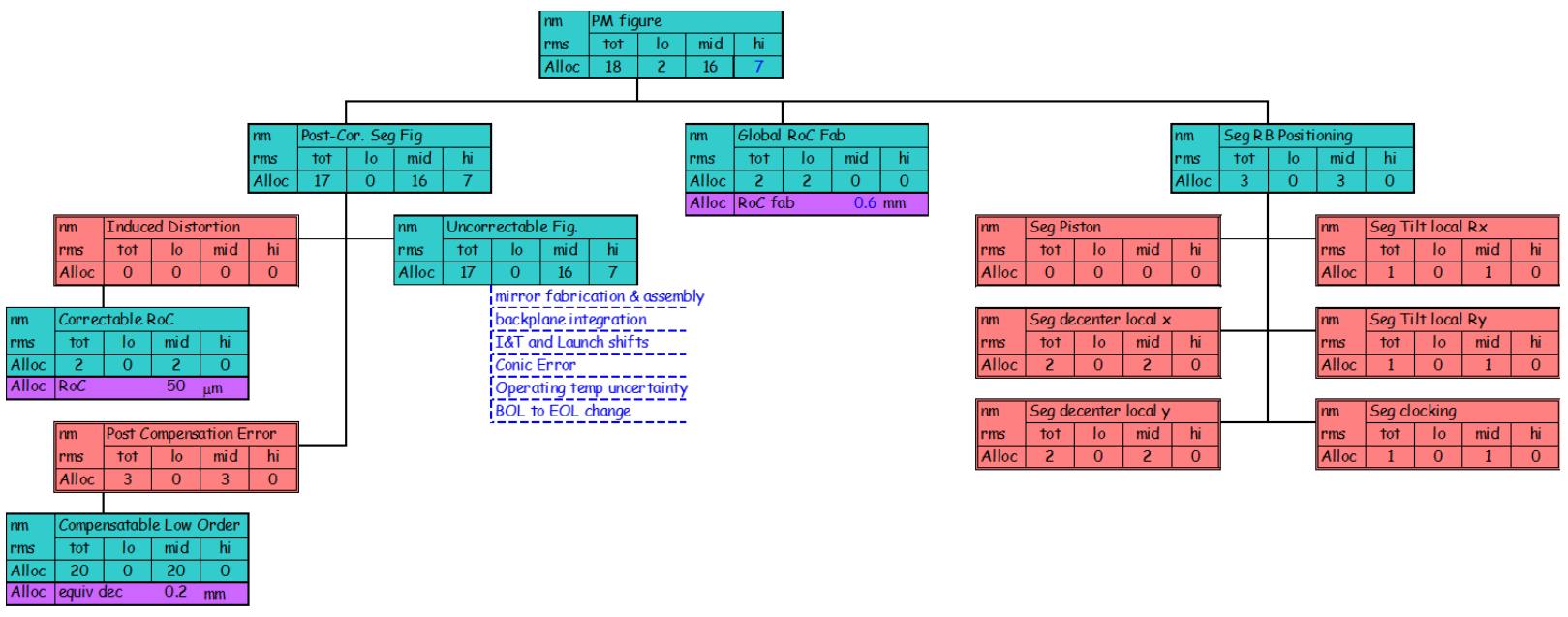

Figure 9. The PM figure is broken into fabrication errors, collective mean RoC fabrication error, and induced figure errors resulting from actuator range compensation for optimizing the 6 dof pose of the segments.

Note that in the special case of coronagraphy, internal DMs in the instrument will compensate some of these higher order errors as part of the process of controlling the PSF speckle to yield high contrast ratios.

The second leg is a small effect associated with RoC. To minimize the errors for the total PM surface, the individual segment RoC are matched to within the WFSC limits to a common value. That common value may not quite be that of the original prescription, but can be compensated by the collective control of the segments with a small residual error.

The final leg is associated with using the 6 dof pose of the individual segments. Not shown in Figure 8 is an underlying starting position error for each segment before WFSC optimization. The amount of motion to correct the starting position errors will induce slight distortions in the surface figure based on the flexure design and are captured in the third leg. These compensation ranges are managed through a separate actuator range budget.

SM, TM, and FSM are not segmented, so their figure budgets reduce to the equivalent single Uncorrectable Figure box shown for the PM. 


\subsection{INSTRUMENTS}

The detailed flow down allocations to the instruments would also follow the traditional separation into design residual, alignment, and fabrication errors. However, at the system level, the aggregate of those errors need to be categorized by calibrated and uncalibrated errors to support the image based WFSC process used for optimizing the telescope. Figure 10 shows the separation and collecting of the error terms needed for WFSC.

The calibrated errors would be based on measured, or verified values, with the uncalibrated portion consisting of the uncertainty in the metrology processes used for calibration combined with the additional wave front change for the integrated instrument as it goes from the calibration environment on the ground to the flight conditions for operations.

As discussed at the beginning of this section (Section 6), the phasing of the PM segments depends on the calibrated measurement of the WFSC controllable modes in the instrument selected for the phasing of the telescope. This is the single field point bias.

Likewise, the alignment of the telescope depends on the calibrated measurement of the field dependent wave forms in each of the instruments. These measurements are combined to determine the multi-field bias.

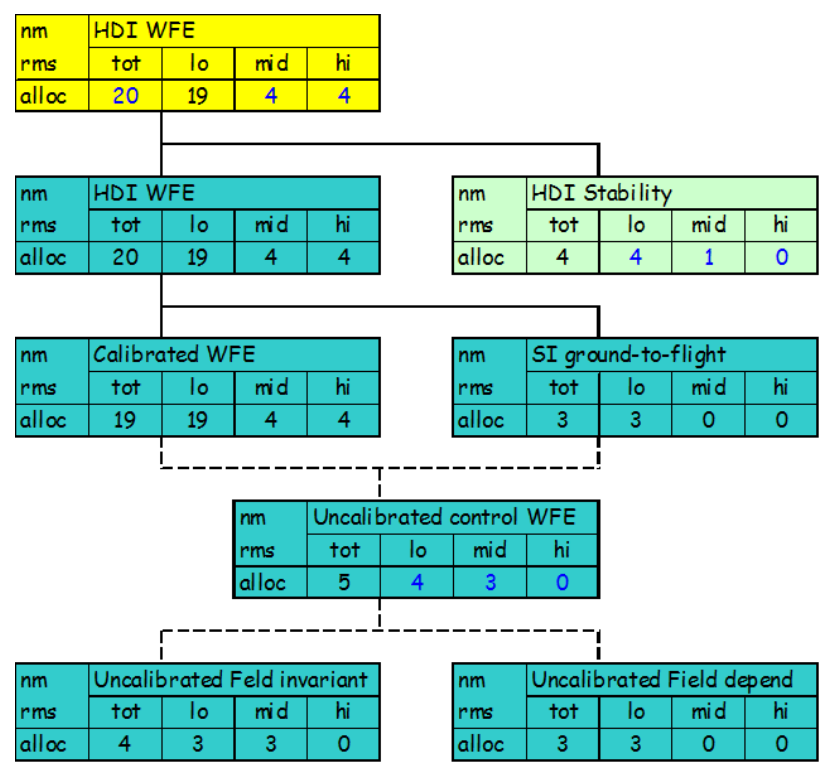

Figure 10. The instrument errors need to be separated into calibrated error, and uncalibrated error. The calibrated wave fronts are used in the WFSC process, and the uncalibrated portion contributes to the sensor bias errors in the WFSC process.

\section{STABILITY}

Different mission goals will have diverse needs for stability, both in spatial frequency content and temporal domains of interest. The two budget drivers to capture the diversity will be radiometric stability for astrophysics observations, and stability of the PSF for high contrast imaging for exoplanet science. Radiometric stability will depend on the stability of the Encircled Energy in a photometric aperture or alternatively, the stability of the image sharpness over time scales of days to months. Stability of a few nm rms WFE will provide long term radiometric stability. Whereas high contrast coronagraphy will depend on maintaining stability of the WF at the spatial frequency of the active controls used to create the dark regions within the PSF over shorter time durations of minutes to hours.

For coronagraphy, active controls are used to shape the PSF to provide the "dark hole" near the core attain high contrast at small inner working angles. The control bandwidth, or time domain, for these controls are limited by the sensing integration times. These controls compensate for errors that are within telescope and coronagraph instrument, affecting the coronagraph imagery. The telescope, and hence other instruments are not affected with the exception that the coronagraph may provide line-of-sight error that is used to stabilize telescope pointing, and hence other instruments. 
Differential imaging may be used to subtract out reference images to improve contrast. This will require wavefront stability over the time intervals used to acquire the science and reference images.

The budget has features to account for the PM sensing and control of segment poses to maintain PM figure, and $\mathrm{PM} / \mathrm{SM}$ sensing and control to maintain PM/SM alignment. The mechanical sensing and compensation to control stability at the telescope level benefits all instruments.

To accommodate these aspects, the stability budget tracks errors in the spatial frequency domains which are in turn allocated into different temporal domains (shown in Figure 11). The passive stability between static WFSC updates are used as the underlying inputs to active control ranges.

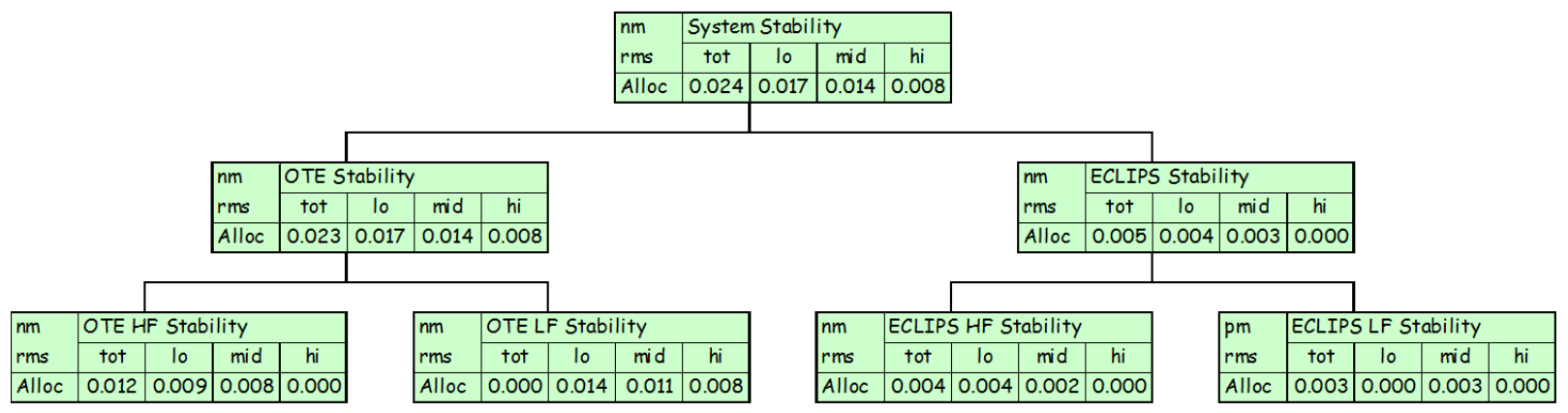

Figure 11. The system stability allocations flow to both the OTE and the instruments which then flow into slow response (LF) and fast response (HF) time domain allocations.

The sub allocations within each time domain flow to components as shown in Figure 12 for the low frequency time domain.

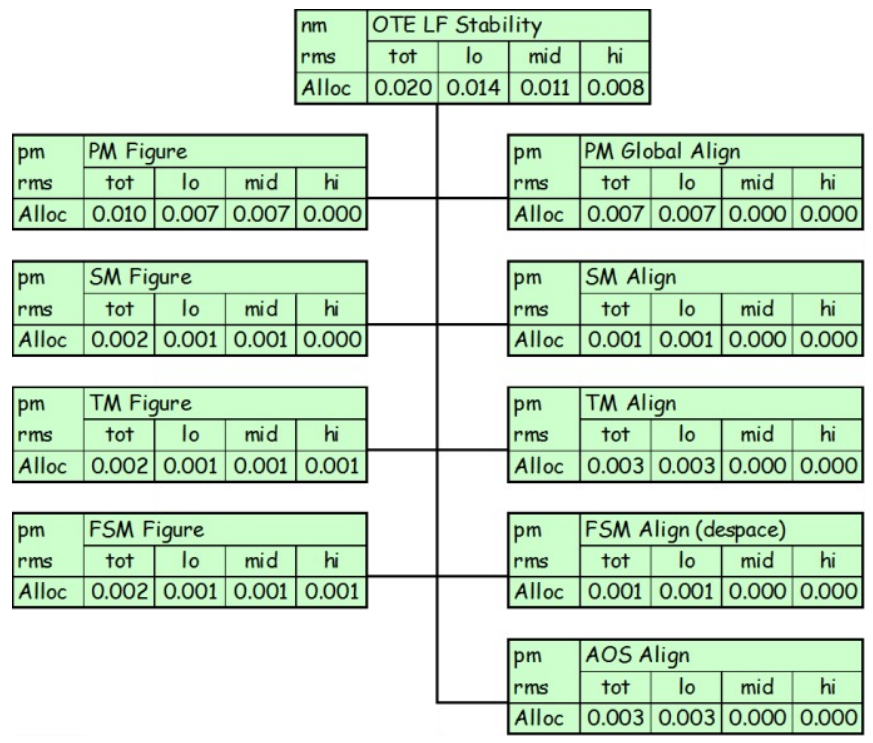

Figure 12. Each of the time domain allocations flow into the component allocations.

\subsection{Example of active thermal control}

An example to show how the budget handles slow response time domain is to look at the example of active thermal control. The example chosen is to look at the despace (piston motion) for a PM segment (Figure 13). A derived linear sensitivity coefficient is used to convert from a WFE allocation to a despace allocation. The despace is sub allocated to deformations of PM segment assembly and the PM backplane support structure. The budget is further distilled into a sensitivity of despace strain to temperature changes, and the temperature control error. These allocations have immediate utility in considering trades between material choices affecting strain sensitivity, design geometry used to 
achieve athermalization, and temperature control methods. The temperature allocation includes allocations to both the sensing noise, and the control error.

The example uses values of $40 \mathrm{~nm} / \mathrm{K}$ strain coefficient and $1 \mathrm{mK}$ tolerances for both the sensing error and control error to illustrate. This would result in $0.226 \mathrm{~nm}$ stability error which is too large for what we want, but may be sufficient to control the compensation range for active mechanical controls. (see next section).

\begin{tabular}{|c|c|c|c|c|c|}
\hline \multirow{2}{*}{$\begin{array}{l}\mathrm{nm} \\
\mathrm{rms}\end{array}$} & \multicolumn{4}{|c|}{ PMSA Despace V1 } & \\
\hline & tot & 10 & mid & hi & \\
\hline alloc & 0.226 & 0.000 & 0.226 & 0 & \\
\hline \multirow[t]{17}{*}{ alloc } & \multicolumn{2}{|c|}{ despace } & \multicolumn{2}{|c|}{$0 \mathrm{~nm}$} & \\
\hline & \multicolumn{5}{|l|}{$\overline{P M S A}$} \\
\hline & alloc & \multicolumn{2}{|c|}{ despace } & \multicolumn{2}{|c|}{$0 \mathrm{~nm}$} \\
\hline & alloc & \multicolumn{2}{|l|}{$n m / K$} & 40 & $\mathrm{~nm} / \mathrm{K}$ \\
\hline & alloc & \multicolumn{2}{|c|}{$\Delta \mathrm{T}$} & 0.001 & $\mathrm{~K}$ \\
\hline & $T(K)$ & \multicolumn{4}{|c|}{ Temp sensing error } \\
\hline & alloc & \multicolumn{2}{|c|}{$\Delta \mathrm{T}$} & 0.00 & $\mathrm{~K}$ \\
\hline & $T(K)$ & \multicolumn{4}{|c|}{ Temp control error } \\
\hline & alloc & \multicolumn{2}{|c|}{$\Delta \mathrm{T}$} & 0.00 & K \\
\hline & \multicolumn{5}{|c|}{ PMBSS } \\
\hline & alloc & \multicolumn{2}{|c|}{ despace } & 0 & $\mathrm{~nm}$ \\
\hline & alloc & \multicolumn{2}{|c|}{$n \mathrm{~m} / \mathrm{K}$} & 40 & $n m / K$ \\
\hline & alloc & \multicolumn{2}{|l|}{$\Delta \mathrm{T}$} & 0.001 & $\mathrm{~K}$ \\
\hline & $T(K)$ & \multicolumn{4}{|c|}{ Temp sensing error } \\
\hline & alloc & \multicolumn{2}{|c|}{$\Delta \mathrm{T}$} & 0.001 & $\mathrm{~K}$ \\
\hline & $T(K)$ & \multicolumn{4}{|c|}{ Temp control error } \\
\hline & alloc & \multicolumn{2}{|c|}{$\Delta \mathrm{T}$} & 0.001 & $\mathrm{~K}$ \\
\hline
\end{tabular}

Figure 13. An example of how the budget goes from WFE into mechanical despace to mechanical strain coefficient and temperature allocations.

\subsection{Example of active mechanical control}

Using the example of section 7.1, the $0.226 \mathrm{~nm}$ rms WFE from despace plus contributions from the other 5 dof alignments and figure stability results in $0.24 \mathrm{~nm}$ rms error from pose stability which is too large for our desires. However, this may be sufficient to maintain the mirrors within the allowable range for active mechanical controls to maintain pose. Figure 14 shows the budget where the result of PMSA figure, backplane stability, and PMSA pose stability are the PM figure input into the active mechanical WFC. The input levels are attenuated by the effectiveness of the control loop which would combine with the mechanical sensing error and pose control error to result in the final figure drift stability. 


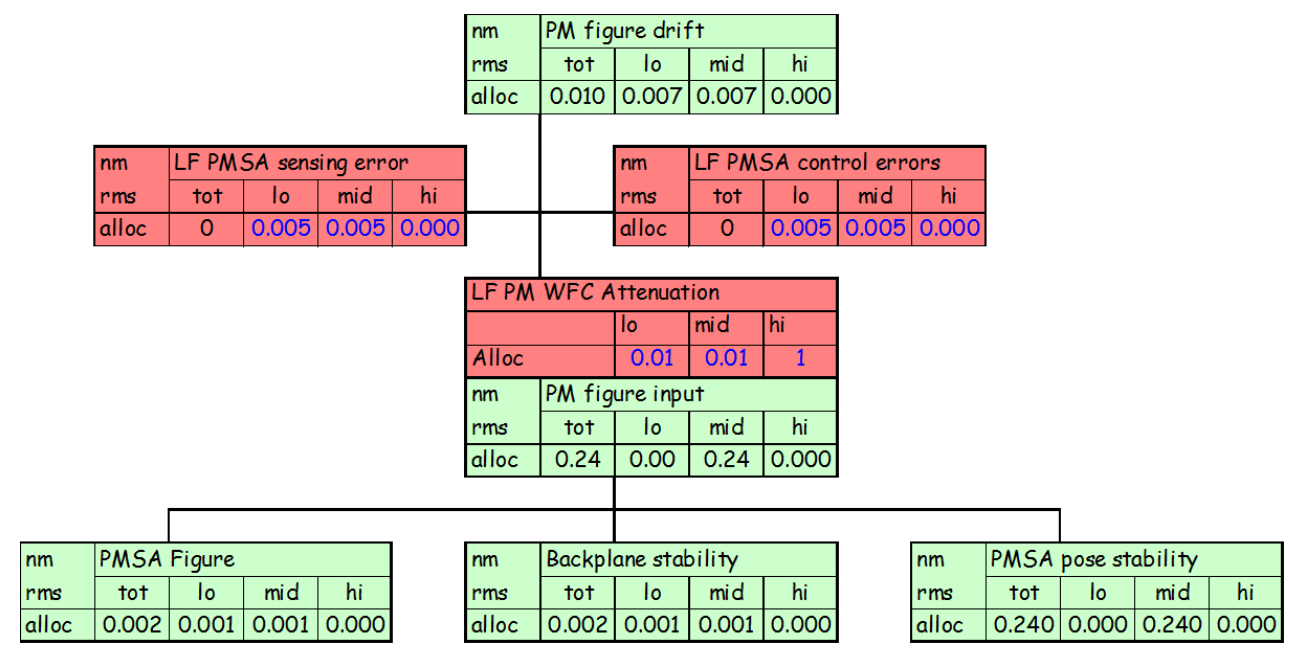

Figure 14. The structure of the budget for active mechanical controls. The input levels are reduced by the attenuation factor of the active controls which then rss with the error signal error and control command error.

\section{IMAGE CENTROID (POINTING) STABILITY}

Motion of the PSF on the image plane during an exposure degrades the final quality of the time integrated image. As discussed previously in Section 3, the Strehl Ratios for WFE and image motion may be used to partition the overall performance budget. There are three forms of motion that are considered in the budget: 1 ) random jitter of the line of sight in orthogonal axes producing a normally distributed Gaussian blur, 2) oscillation of the image along a linear direction, and 3) a constant rate motion long a linear direction. The first case is produced in response to dynamic disturbance in the system and residual noise in the system pointing control. The second case is produced by the residual noise in the roll control of the system producing small oscillating roll of the field of view about the guide star line of sight. This motion will cause other targets in the field to move along short lengths of arcs about the anchored field point of the guide star. This effect can be approximated as a linear motion. The third case is for slower thermal distortions in the system that cause relative drift in the boresight of instruments relative to the guider instrument, or systematic roll of the telescope relative to the axes of the roll control sensing system.

The budget allocation for image motion is divided into three domains: motion that occurs at temporal frequencies above the control bandwidth of the fine guidance control, the unrejected error from the fine guidance control performance, and motions that are not sensed by the fine guidance system. Each domain is shown as a leg in the budget shown in Figure 15.

The line of sight response to dynamics above the control bandwidth will be a composite of response functions and treated as Gaussian blurs. Integrated modeling of the system that includes the sources of dynamic disturbance and factors that isolate the transmittance of the disturbance into the optical system response is used to inform this portion of the budget. The factors in the model include structural stiffness, damping, and active isolation control.

The unrejected line of sight motion within the guidance control bandwidth has three components. The first is the unrejected error in the two-axis line of sight fine guidance control. The second the component that accounts for the unrejected roll control that produces the field rotation motion. And the third is the result of optical field distortion combined with using a fine steering mirror (FSM) to compensate telescope boresight motion. This differential distortion results in the motion of targets at field locations separated from the guide star location causing an induced smear. The amount of smear is proportional to the magnitude of FSM compensation and can be reduced by off-loading the FSM to the outer gimbal control. Alternatively, the telescope design could be modified with a fourth mirror to reduce the optical distortion, but at a performance cost in transmission (important in the UV) and added system complexity.

The third leg in the budget is for those motions not sensed by the fine guidance system. These correspond to structural deformations between the guidance reference frame and the instruments. There are two aspects. The first is internal structural deformations between the optical alignment of the fine guide sensor and the other instruments. These will 
depend principally on thermal stability of the system. The second is structural deformations that cause the whole system to roll in field relative to the reference coordinate system for the roll control sensors. This will be dependent on the structural paths between the Attitude Control System star trackers and the telescope if relying on ACS control, or between multiple guide star trackers located on the telescope.

Applicable to the first leg, and to the earlier discussions in Section 7 on stability of the optics, there is conceptually five opportunities to control and reduce the impacts of the dynamic disturbances. The first is to reduce or eliminate the disturbance source levels. For LUVOIR, disturbances from the three-axis attitude control system will be the area of interest. The second opportunity is to dynamically isolate the source from the system. This can be by either passive or active techniques. The third opportunity is to design structural links between systems that assist in reducing transmittance of the disturbance. The fourth is to isolate the payload (telescope and instruments). And the fifth is to use active compensation at the payload such as active line of sight control with the FSM or active sensing and control of mirror poses.

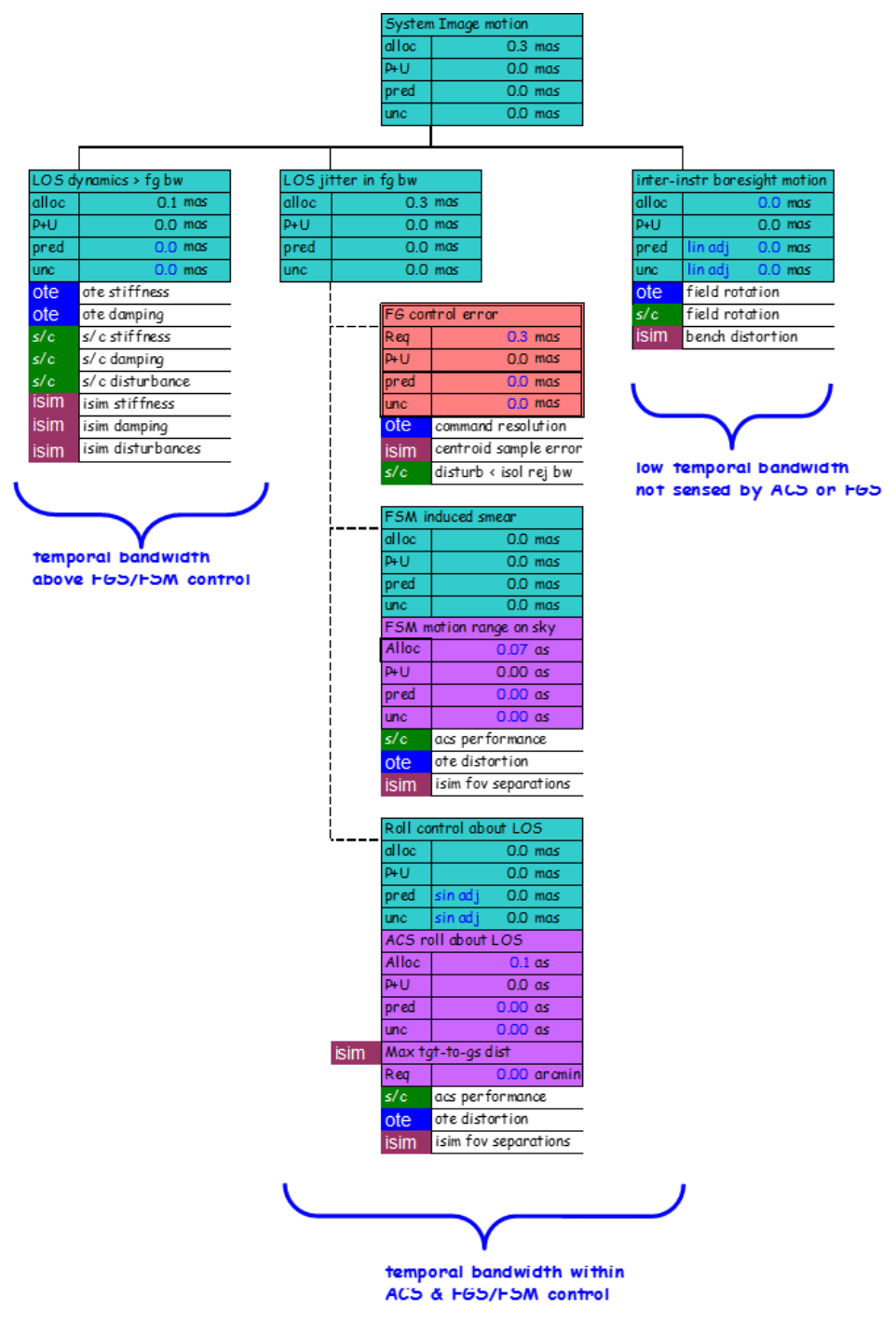

Figure 15. The image motion contribution to the budget is divided into three legs: motion above the active fine guidance control bandwidth; the unrejected error within the control bandwidth; and motions not sensed by the fine guidance system. 


\section{SUMMARY}

In summary, large space telescopes offer increased angular resolution and signal collection capabilities, but these larger sizes require post launch deployment with remote alignment and phasing after launch. An optical budget methodology was developed for JWST to account for the active deployment and in-flight control aspects. The methodology uses a staged approach to account for the layers of active control. That is, budgeting for the conditions to be corrected/compensated as well as the output following active control. Features of a Segmented PM system shift the distribution of errors to a higher weighting in the mid and high spatial frequency content, putting more emphasis on Encircled Energy compared to Strehl Ratio. The use of science instruments for the WFSC process does lead to interaction of instrument wavefront errors through sensor bias errors that is included in the budget accounting.

This methodology has been expanded to be applicable to LUVOIR. High contrast imaging using coronagraphy adds another layer to the staged approach to the budgeting. Coronagraphy drives both the high precision control of mid spatial frequencies in the instrument and tight levels of temporal stability for the whole telescope and instrument. These tight stability requirements will drive adding temporal bandwidth capabilities to actuators, system level thermal design and control considerations, and system level vibration controls.

The challenges are tightening the overall budget allocations to account for going from a diffraction limited wavelength of $2000 \mathrm{~nm}$ to $500 \mathrm{~nm}$, and going from stability requirements of $\approx 50 \mathrm{~nm}$ rms over periods of days to weeks, to picometer stability levels over minutes to hours. This drives reduction in mirror figure errors, actuator resolution, and line of sight motion.

Technologies exist that can be used to evolve from JWST designs into a configuration that can meet these more stringent performance levels ${ }^{8,9,10,11}$.

\section{ACKNOWLEDGEMENTS}

We acknowledge the support and contributions of the LUVOIR team and the many engineers and scientist among the many organizations that make up the astrophysics space telescope community for the many discussions and interchanges on these topics. 


\section{REFERENCES}

[1] Julie A. Crooke, et. al., "Status and path forward for the large ultraviolet/optical/infrared surveyor (LUVOIR) mission concept study”, Proc. SPIE, 9904 (2016)

[2] Matthew R. Bolcar, et. al., "Initial Technology Assessment for the Large-Aperture UV-Optical Infrared (LUVOIR) Mission Concept Study”, Proc. SPIE, 9904 (2016)

[3] Marc Postman, et. al. "Advanced Technology Large-Aperture Space Telescope: A technology roadmap for the next decade", A NASA Astrophysics Strategic Mission Concept Study, Space Telescope Science Institute, May 2009

[4] Paul A. Lightsey and Michael Chrisp, "Image Quality for large segmented space telescopes”, Proc. SPIE, 4850 (2002)

[5] Mast, T. S., Nelson, J. E., Welch, W. J., "Effects of primary mirror segmentation on telescope image quality", International Conference on Advanced Technology Optical Telescopes, 332, p.123-133, SPIE, Bellingham, WA, 1982.

[6] Wetherell, W. B., Applied Optics and Optical Engineering, Vol. VIII, Chap. 6, Academic Press, New York, NY 1980.

[7] Bijan Nemati, et. al., "The effects of space telescope primary mirror segment errors on coronagraph instrument performance," Proc. SPIE 10398, 103980G (5 September 2017); doi: 10.1117/12.2273072

[8] Matthew R. Bolcar, et. al., "The Large UV/Optical/Infrared (LUVOIR) Surveyor: Decadal Mission concept design update”, Proc. SPIE, 10398 (2017)

[9] Matthew R. Bolcar, et. al., "The Large UV/Optical/Infrared (LUVOIR) Surveyor: Decadal Mission concept technology development overview”, Proc. SPIE, 10398 (2017)

[10] Lee D. Feinberg, et.al., "Ultra-stable segmented telescope sensing and control architecture", Proc. SPIE, 10398 (2017)

[11] Arnold A. Barnes III, et. al., “Technology advancements for future astronomical missions”, Proc. SPIE, 10398 (2017) 